\title{
Mean flow anisotropy without waves in rotating turbulence
}

\author{
Jonathan A. Brons ${ }^{1,2, \dagger}$, Peter J. Thomas ${ }^{2}$ and Alban Pothérat ${ }^{1}$ \\ ${ }^{1}$ Fluid and Complex Systems Research Centre, Coventry University, Coventry CV1 5FB, UK \\ ${ }^{2}$ Fluid Dynamics Research Centre, School of Engineering, University of Warwick, \\ Coventry CV4 7AL, UK
}

(Received 23 August 2019; revised 30 January 2020; accepted 31 January 2020)

We tackle the question of how anisotropy in flows subject to background rotation favours structures elongated along the rotation axis, especially in turbulent flows. A new, wave-free mechanism is identified that challenges the current understanding of the process. Inertial waves propagating near the rotation axis (Staplehurst et al. $J$. Fluid Mech., vol. 598, 2008, pp. 81-105; Yarom \& Sharon, Nat. Phys., vol. 10(7), 2014, pp. 510-514) are generally accepted as the most efficient mechanism to transport energy anisotropically. They have been shown to transfer energy to large anisotropic, columnar structures. Nevertheless, they cannot account for the formation of simpler steady anisotropic phenomena such as Taylor columns. Here, we experimentally show that more than one mechanism involving the Coriolis force may promote anisotropy. In particular, in the limit of fast rotation, that is at low Rossby number, anisotropy favouring the direction of rotation of the average of a turbulent flow arises neither because of inertial waves nor following the same mechanism as in steady Taylor columns, but from an interplay between the Coriolis force and average advection.

Key words: rotating turbulence, waves in rotating fluids

\section{Introduction}

Subjecting a flow to background rotation tends to eliminate variations of velocity along the axis of rotation. The effect, first noticed by Lord Kelvin (Thomson 1868), was famously illustrated when Taylor observed that a fluid column exactly followed the motion of a coin placed at the bottom of a rotating tank (Taylor 1922). The question of the anisotropic mechanism underlying the development of these columnar structures is, however, still open. It raises the wider issue of how anisotropy favouring the direction of rotation arises in rotating flows, which is the focus of this work. The thesis we advocate is that non-propagative mechanisms can promote anisotropy, in particular purely advective ones.

The type of anisotropy that underpins the emergence of Taylor columns in rotating flows is most commonly studied in the context of turbulence in fast rotating systems

$†$ Email address for correspondence: jonbrons@gmail.com 
such as planetary cores, atmospheres and astrophysical objects, where its origin is attributed to the propagation of inertial waves (Hopfinger, Browand \& Gagne (1982) and Davidson (2013), and see Greenspan (1968) for the theory of these waves): for a wavevector $\boldsymbol{k}$ of frequency $\omega$, in background rotation $\boldsymbol{\Omega}$, inertial waves follow the dispersion relation, and associated group velocity

$$
\omega= \pm 2 \boldsymbol{\Omega} \cdot \boldsymbol{e}_{k}, \quad \boldsymbol{v}_{g}= \pm \frac{2}{k} \boldsymbol{e}_{k} \times\left(\boldsymbol{\Omega} \times \boldsymbol{e}_{k}\right)
$$

where $\boldsymbol{e}_{k}=(1 / k) \boldsymbol{k}$. Two main theories account for the growth of anisotropy favouring the direction of rotation and the subsequent spontaneous formation of large structures in these systems: one invokes triadic interactions feeding an inverse energy cascade towards large scales while non-resonant triads or quartets of waves transfer energy to modes aligned with the axis of rotation (Cambon \& Scott 1999; Smith \& Waleffe 1999). This scenario is supported by numerical simulations and by strong experimental (Lamriben, Cortet \& Moisy 2011; Duran-Matute et al. 2013; Campagne et al. 2014) and numerical (Smith, Chasnov \& Waleffe 1996; Chen et al. 2005) evidence of the inverse energy cascade. The other theory argues that linear inertial waves account for most of the energy transport in rotating turbulence (Davidson, Staplehurst \& Dalziel 2006). This was demonstrated numerically and experimentally in the context of the propagation of transient rotating turbulence (Screenivasan \& Davidson 2008; Staplehurst, Davidson \& Dalziel 2008; Kolvin et al. 2009).

Both theories consider Taylor columns as large turbulent scales, that is, as transient, fluctuating objects. None of them, however, satisfactorily explains the steady, laminar columns that Taylor observed. Indeed, the analytical solution for these columns (Moore \& Saffman 1969) is entirely steady and neglects non-rotating inertia, thus excluding inertial waves. The formation of fluctuating Taylor columns in rotating turbulent flows and steady ones in laminar flows may thus arise out of fundamentally different mechanisms. This idea finds support in previous experimental and theoretical work (McEwan 1973, 1976; Pothérat 2012), showing that a velocity field with a divergence in planes normal to the rotation preferentially transports momentum along the rotation axis. Crucially, this phenomenology holds regardless of the steady or fluctuating nature of the flow. We therefore suggest that more than a single mechanism may exist to promote anisotropy in rotating flows and set out to determine conditions in which the better known mechanisms involving inertial waves may not be dominant. Beyond simple steady flows, we seek evidence of such alternative mechanism not involving inertial waves in the average components of turbulent flows, on the grounds that these are both steady in nature and subject to the presence of inertial waves inherent to rotating turbulence. As such, they provide the ideal battleground for mechanisms with and without waves to compete. The particular flow of interest shall be generated in an experimental device where turbulent motion results from the fast injection of fluid through holes located at the bottom of a cylindrical tank driven in rotation. This technique, pioneered by McEwan (1973), provides an efficient and convenient mean of generating turbulence with a background rotation (see more recent experiments by Kolvin et al. (2009)). For the specific purpose of the study, using a small number of holes enables us to imprint a strong average component to the turbulence.

We first establish a scaling law for the length scale of anisotropic structures forming out of momentum transport associated with average flows with a divergence in planes normal to the rotation $(\S 2)$. The experimental set-up and optical measurement 
techniques based on particle image velocimetry (PIV) are detailed in $\S 3$. We then proceed in three steps gathered in $\S 4$ : first, the theoretical scaling for the length scale of columnar structures is validated against experimental measurements. Then, in order to separate the contribution of random fluctuations from that of inertial waves to the topology of the average flow, we introduce a filtering technique using the maximum frequency of inertial waves. Based on this technique, we derive mathematical upper bounds for terms involving inertial waves in the equation governing the average velocity field. The relevance of these upper bounds is tested by means of more refined filtering techniques inspired by Yarom \& Sharon (2014). Finally we derive an equation for the average flow and experimentally estimate upper bounds for terms involving inertial waves to identify flow regimes where the contributions of these waves in this equation can be neglected. The implications of these results are discussed in $\S 5$.

\section{Theory}

\subsection{Governing equations and diffusion length scale}

We first derive scalings characterising anisotropy between the rotation axis and other directions in steady and turbulent flows. Consider an incompressible flow of Newtonian fluid in a frame of reference rotating at constant angular velocity $\Omega \boldsymbol{e}_{z}$. The effect of the Coriolis force on a structure of size $l_{z}$ along the axis of rotation, $l_{\perp}$ in the directions perpendicular to it and velocity $U$ is readily seen from the $z$-component of the vorticity equation governing the velocity and vorticity fields $\boldsymbol{u}$ and $\boldsymbol{\omega}$

$$
\left(\frac{\mathrm{d}}{\mathrm{d} t}-v \Delta\right) \omega_{z}=\omega \cdot \nabla u_{z}+2 \Omega \partial_{z} u_{z},
$$

where, $\mathrm{d} / \mathrm{d} t=\partial_{t}+\boldsymbol{u} \cdot \nabla$. In the limit $\Omega \rightarrow \infty$ the flow is columnar, with $\partial_{z} u_{z}=0$, which implies $-\nabla_{\perp} \cdot \boldsymbol{u}_{\perp}=0$. For finite rotation, a horizontally divergent flow exists and the Coriolis force associated with it must be balanced either by inertial or viscous forces (Pothérat 2012). The divergent flow is estimated by means of the $z$-component of the momentum equation and its divergence, i.e.

$$
\begin{gathered}
\left(\frac{\mathrm{d}}{\mathrm{d} t}-v \Delta\right) u_{z}=-\partial_{z} \frac{p}{\rho}, \\
\nabla \cdot(\boldsymbol{u} \cdot \nabla \boldsymbol{u})=2 \Omega \omega_{z}-\Delta \frac{p}{\rho} .
\end{gathered}
$$

In both Taylor's experiment (Taylor 1922) and Moore \& Saffman's analytical solution (Moore \& Saffman 1969), inertia is neglected. In this limit (2.3) implies that the pressure is geostrophic $p=2 \rho \Omega \Delta^{-1} \omega_{z}$, where the inverse of the Laplacian $\Delta^{-1}$ is defined with boundary conditions prescribed by the geometry. The rotational part of the Coriolis force can thus be expressed by virtue of (2.2) as

$$
2 \Omega \partial_{z} u_{z}=4 \frac{\Omega^{2}}{v} \partial_{z z}^{2} \Delta^{-2} \omega_{z} .
$$

An almost identical mathematical form exists for the Lorentz force in electrically conducting fluids pervaded by an imposed magnetic field $B \boldsymbol{e}_{z}$, where it expresses that the Lorentz force diffuses momentum along $\boldsymbol{e}_{z}$ (Sommeria \& Moreau 1982). 
This finding was experimentally verified, establishing that the diffusive nature of the Lorentz force persists both in viscous and inertial regimes, albeit with different characteristic diffusion length scales (Sommeria \& Moreau 1982; Pothérat \& Klein 2014; Baker et al. 2018). In the rotating flows of interest here, the Coriolis force diffuses momentum along $\boldsymbol{e}_{z}$ in the inertialess limit. Its diffusion length scale follows from introducing (2.4) into (2.1) and applying scaling arguments

$$
l_{z}^{v}\left(l_{\perp}\right) \sim l_{\perp} \frac{2 \Omega l_{\perp}^{2}}{v}=l_{\perp} \frac{l_{\perp}^{2}}{H^{2}} E^{-1},
$$

where the Ekman number $E=v / 2 \Omega H^{2}$ represents the ratio of Coriolis to viscous forces, based on the domain height $H$ (cf. figure 1). This length scale recovers the columnar length scale implied in Moore \& Saffman's (1969) analytical solution. $l_{z}^{v}$ can be interpreted as the distance needed for viscous effects to exhaust the horizontally divergent flow that drives the column.

In contrast to Taylor's flow (Taylor 1922), inertia dominates in turbulent flows and balances the Coriolis force associated with the horizontally divergent flow in (2.1). Using this assumption and a similar derivation as for $l_{z}^{\nu}$ leads to an inertial scaling for $l_{z}$

$$
l_{z}^{I}\left(l_{\perp}\right) \sim \frac{2 \Omega l_{\perp}^{2}}{U}=l_{\perp} R o\left(l_{\perp}\right)^{-1},
$$

where the Rossby number $R o\left(l_{\perp}\right)=U / 2 \Omega l_{\perp}$ is based on the considered structure's velocity scale $U$. It represents the ratio of inertial to Coriolis forces at the scale of the structure considered.

\subsection{Influence from the fluctuating flow on the average flow component}

To isolate the mechanisms controlling anisotropy, we consider a forced, anisotropic turbulent flow with non-zero average flow at large Reynolds number. A benefit of this choice of flow is that mechanisms controlling the anisotropy of the average flow that do not involve waves, as in Taylor columns, can be captured by simple event averaging. At the same time, since turbulent fluctuations under strong rotation support inertial waves, these can potentially affect the anisotropy of the average flow. For these reasons, a turbulent flow with an average flow component offers a good testing ground to identify the conditions in which either propagative or wave-free mechanisms drive anisotropy. We start by deriving the equations for the average quantities, decomposing all quantities into their average and fluctuations, e.g. $\boldsymbol{u}=\langle\boldsymbol{u}\rangle+\boldsymbol{u}^{\prime}$. Taking the average of (2.1)-(2.3), neglecting viscous friction yields

$$
\begin{gathered}
\langle\boldsymbol{u}\rangle \cdot \nabla\left\langle\omega_{z}\right\rangle=\langle\boldsymbol{\omega}\rangle \cdot \nabla\left\langle u_{z}\right\rangle+2 \Omega \partial_{z}\left\langle u_{z}\right\rangle+\left\langle\boldsymbol{\omega}^{\prime} \cdot \nabla u_{z}^{\prime}\right\rangle-\left\langle\boldsymbol{u}^{\prime} \cdot \nabla \omega_{z}^{\prime}\right\rangle, \\
\langle\boldsymbol{u}\rangle \cdot \nabla\left\langle u_{z}\right\rangle=-\partial_{z} \frac{\langle p\rangle}{\rho}-\left\langle\boldsymbol{u}^{\prime} \cdot \nabla u_{z}^{\prime}\right\rangle, \\
\Delta \frac{\langle p\rangle}{\rho}=2 \Omega\left\langle\omega_{z}\right\rangle-\nabla \cdot\langle\boldsymbol{u} \cdot \nabla \boldsymbol{u}\rangle .
\end{gathered}
$$

In (2.9), $|\nabla \cdot\langle\boldsymbol{u} \cdot \nabla \boldsymbol{u}\rangle| /\left|\Omega\left\langle\omega_{z}\right\rangle\right|=O(R o)$, so for fast rotating turbulence $(R o \ll 1)$, the average pressure is mostly governed by a geostrophic balance

$$
\frac{\langle p\rangle}{\rho}=2 \Omega \Delta^{-1}\left\langle\omega_{z}\right\rangle+O\left(U^{2} R o\right) .
$$




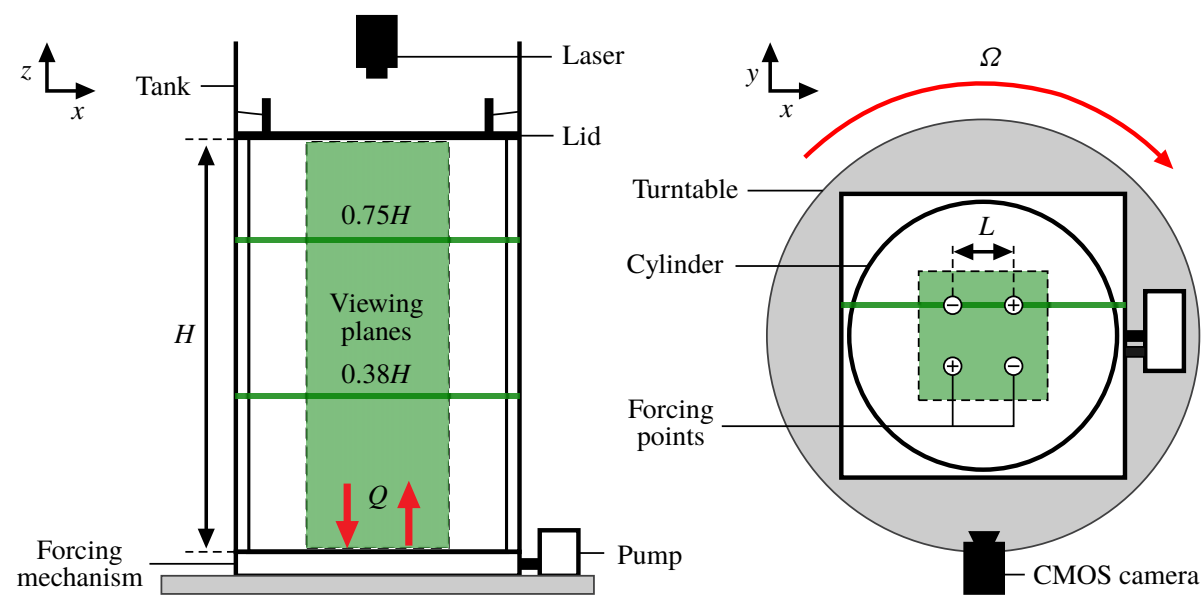

FIGURE 1. Side- and top-view sketch of the experimental set-up. Green regions and lines show areas and positions of PIV planes used during measurements. In top-view $(+)$ refers to a source and (-) to a sink.

Scaling arguments do not permit us to further simplify (2.7), (2.8). The reason is that, since columnar structures are far longer than wide $\left(l_{z}^{I} \gg l_{\perp}\right)$, $z$-derivatives can be approximated as $\partial_{z} \sim\left(l_{z}^{I}\right)^{-1}$, implying that all terms in (2.8) are $O\left(U^{2} / l_{\perp}\right)$ and all terms in (2.7) are $O\left(U^{2} / l_{\perp}^{2}\right)$. The potential influence of fluctuations on the anisotropy of the average flow can, however, be analysed by experimentally evaluating the magnitude of all the terms in (2.7) and (2.8). Of particular interest are the last two terms in (2.7) and the last term in (2.8) as fluctuations and thus inertial waves, can only affect the average flow through them.

\section{Experimental methods}

\subsection{Experimental apparatus}

The experimental set-up consists of a rectangular tank $(600 \mathrm{~mm} \times 320 \mathrm{~mm} \times 320 \mathrm{~mm})$ fitted at the centre of a rotating turntable. The flow is forced by injecting and subtracting fluid through four holes (diameter $d=1 \mathrm{~mm}$ ) located at the corners of a $L=53 \mathrm{~mm}$ square in the bottom wall of the tank (figure 1). All holes are connected to a peristaltic pump simultaneously injecting fluid through holes along one diagonal of the square and sucking fluid through the others, at the same constant flow rate $Q$ through each hole. This configuration was chosen to both cancel the injected mass flux, and to minimise the displacement of vortices forming along the subtraction and injection jets. A cylinder (height $H=400 \mathrm{~mm}, \varnothing 300 \mathrm{~mm}$ ) closed by an upper transparent lid placed inside the tank prevents free-surface deformation and provides a viewing window for the optical measurements. The set-up is spun up into solid body rotation at a rotation speed $\Omega$, before the pump is initiated. Prior to measurements, the flow is left to settle into a statistically steady state. Statistical steadiness was assessed through the convergence of statistical quantities, kept below $1 \%$ in most cases.

The governing parameters are the Ekman number $E=v / 2 \Omega H^{2}$ and a forcing-based Reynolds number, $R e_{Q}=4 Q / \pi v d$. They are independently controlled by $\Omega$ and $Q$. The flow intensity is measured a posteriori by means of average- and a fluctuationbased Rossby numbers $R o=\langle|\boldsymbol{u}|\rangle_{x t} / 2 \Omega L$ and $R o^{\prime}=\left\langle\left|\boldsymbol{u}^{\prime 2}\right|\right\rangle_{x t}^{1 / 2} / 2 \Omega L$, built on time and 
space averages $\langle\cdot\rangle_{x t}$ over the horizontal visualisation plane at $z=0.75 \mathrm{H}$. Experiments are performed over a range of parameters spanning $E=\{4.25,5.67,8.59,17,34\} \times$ $10^{-5}$ and $3 \times 10^{2} \leqslant R e_{Q} \leqslant 1.5 \times 10^{4}$. In this range, the jets penetrating the flow are always turbulent. Velocity fields are measured with a two-dimensional (2D) PIV system: a laser sheet illuminates horizontal planes (HP) at $z=0.38 \mathrm{H}$ or $z=0.75 \mathrm{H}$, or a vertical plane (VP) aligned on a injection/subtraction pair. For visualisations in the $\mathrm{HP}$, a 1.3MP CMOS camera records a $150 \mathrm{~mm} \times 150 \mathrm{~mm}$ area centred on the tank at 30 fps. For the VP experiments, two cameras record an area of $400 \mathrm{~mm} \times 150 \mathrm{~mm}$ at $60 \mathrm{fps}$ along the tank. The smallest resolvable distance is $2.1 \mathrm{~mm}$ in all planes.

\subsection{Evaluating the average flow quantities}

Evaluating $\left\langle\boldsymbol{\omega}^{\prime} \cdot \nabla u_{z}^{\prime}\right\rangle,\left\langle\boldsymbol{u}^{\prime} \cdot \nabla \omega_{z}^{\prime}\right\rangle$ and $\left\langle\boldsymbol{u}^{\prime} \cdot \nabla u_{z}^{\prime}\right\rangle$, requires us to calculate expressions such as $\partial_{z} \omega_{z}$ that are not directly accessible from 2D-PIV data. It is, however, possible to calculate rigorous upper bounds for these three-dimensional quantities, using the two-dimensional quantities accessible through plane PIV measurements only. We start by noticing that the symmetry of the forcing and the geometry imply that statistical properties are invariant by rotation of $\pi / 2$ followed by a symmetry with respect to any vertical plane equidistant from two fluid injection/subtraction points located on any one side of the square they form. Hence statistical properties in the $x$ and $y$ directions are identical, so that

$$
\left|\left\langle\boldsymbol{u}^{\prime} \cdot \nabla u_{z}^{\prime}\right\rangle\right|=\left|\left\langle u_{z}^{\prime} \partial_{z} u_{z}^{\prime}\right\rangle\right|+2\left|\left\langle u_{x}^{\prime} \partial_{x} u_{z}^{\prime}\right\rangle\right|=\left|\left\langle\boldsymbol{u}^{\prime} \cdot \nabla u_{z}^{\prime}\right\rangle\right|^{e} .
$$

Here, the superscript ${ }^{e}$ refers to the estimates for the three-dimensional terms that we built out of quantities that we actually measured with the 2D-PIV system. An upper bound estimate is obtained for $\left|\left\langle\omega^{\prime} \cdot \nabla u_{z}^{\prime}\right\rangle\right|$, using Schwartz's inequality to bound the average of products with the product of averages, Minkowsky's inequality to handle sums and, again, statistical equivalence of directions $x$ and $y$

$$
\left|\left\langle\omega^{\prime} \cdot \nabla u_{z}^{\prime}\right\rangle\right| \leqslant 2\left\langle\left|\omega_{y}^{\prime}\right|^{2}\right\rangle^{1 / 2}\left\langle\left|\partial_{x} u_{z}^{\prime}\right|\right\rangle^{1 / 2}+\left\langle\left|\omega_{z}^{\prime}\right|^{2}\right\rangle^{1 / 2}\left\langle\left|\partial_{z} u_{z}^{\prime}\right|^{2}\right\rangle^{1 / 2}=\left|\left\langle\omega^{\prime} \cdot \nabla u_{z}^{\prime}\right\rangle\right|^{e} .
$$

In the resulting estimate, all terms are evaluated from VP-PIV except $\left\langle\left|\omega_{z}^{\prime}\right|^{2} \mid\right\rangle^{1 / 2}$, which is obtained from HP-PIV. Using a similar approach, an upper bound estimate is obtained for the reminding three-dimensional term as

$$
\left|\left\langle\boldsymbol{u}^{\prime} \cdot \nabla \omega_{z}^{\prime}\right\rangle\right| \leqslant\left|\left\langle u_{x}^{\prime} \partial_{x} \omega_{z}^{\prime}\right\rangle+\left\langle u_{y}^{\prime} \partial_{y} \omega_{z}^{\prime}\right\rangle\right|+\left\langle\left|u_{z}^{\prime}\right|^{2}\right\rangle^{1 / 2}\left\langle\left|\partial_{z} \omega_{z}^{\prime}\right|^{2}\right\rangle^{1 / 2}=\left|\left\langle\boldsymbol{u}^{\prime} \cdot \nabla \omega_{z}^{\prime}\right\rangle\right|^{e} .
$$

Additionally, contributions from inertial waves to these terms are estimated by filtering out velocity and vorticity components whose frequency exceeds the maximum possible frequency of inertial waves, $2 \Omega$ (Greenspan 1968). An upper bound for the contribution of inertial waves is obtained by treating all remaining fluctuations in terms filtered in this way as if they were inertial waves. As such, $\left\langle\left|\partial_{z} \omega_{z}^{\prime}\right|^{2}\right\rangle^{1 / 2}$ is estimated by replacing $\partial_{z}$ by an upper estimate $V_{I}(H) \partial_{t}$, where $V_{I}(H)$ is the fastest inertial wave group velocity, i.e. that associated with the largest possible scale in the vessel, $H$.

To find an upper bound for the contribution of inertial waves to estimates for the nonlinear terms derived in $\$ 3.2$, each of the measured velocity and fields are split in their two components $f>2 \Omega$ and $f<2 \Omega$. As such, nonlinear terms express as four components, only one of which is exclusively built from wave-free velocity contributions. The remaining three components form the upper bound for the 
contribution of inertial waves to the nonlinear term. Spatial derivatives are calculated by means of a fourth-order centred finite difference scheme.

We stress again here that the derivation of these upper bounds requires no simplifying assumption, other than assuming sufficient statistical convergence of our dataset to achieve statistical equivalence of properties along $x$ and $y$. As such these upper bounds require no validation, as long as they are handled as such. In particular, to show that the actual three-dimensional terms are negligible compared to the contribution of the Coriolis force, it is sufficient to show that the two-dimensional (2-D) upper bounds are, without further validation. The flip side of this approach is that it is difficult to assess how close estimates are from the actual values. Consequently, even if the estimates are not found significantly smaller than the contribution of the Coriolis force, the actual three-dimensional quantities may still be.

\section{Results}

For the range of parameters we consider, turbulent jets form above the two injection/subtraction points and feed a small turbulent patch dominated by inertia rather than by the Coriolis force. This patch extends to a critical height $h_{p}$ such that the local Rossby number at $h_{p}$ reaches unity. A similar patch exists in rotating grid turbulence (Dickinson \& Long 1983) and in turbulent convective plumes under the effect of rotation (Maxworthy \& Narimousa 1994). A more detailed analysis of this region can be found in Brons, Thomas \& Pothérat (2020). Columnar structures develop above the patch where $z>h_{p}$. The lower limit $\left(R o<10^{-2}\right)$ coincides with a regime of weaker turbulence, dominated by a quadrupole of robust columnar structures, reminiscent of the 2-D flow solutions identified by Gallet (2015) when rotating flows become two-dimensional. The quadrupole sits above the turbulent patch and is aligned with the 4 injection/subtraction holes. At higher Ro, the columns unlock from the forcing points and become subject to mutual advection, pairing and merging (Tabeling 2002).

\subsection{Columnar length scale in forced rotating turbulence}

We start by confronting the scalings for the vertical length scales (2.6) to experimental data. We build two vertical length scales $l_{z}$ and $l_{z}^{\prime}$ from experiments along the vertical plane using two-point velocity correlations $C_{u_{x}}(\delta z)$ and $C_{u_{x}^{\prime}}(\delta z)$, respectively calculated from the full velocity field $u_{x}$ or its fluctuating part $u_{x}^{\prime}$ (Aujogue et al. 2018), using

$$
C_{u_{x}}(\delta z)=\left\langle\int_{A} u_{x}(x, z+\delta z) u_{x}(x, z) \mathrm{d} x \mathrm{~d} z\right\rangle_{t} .
$$

Here, $A$ is the area of the flow field captured by the measurements along the vertical plane, $\langle\cdot\rangle_{t}$ is a time average and $\delta z$ is the separation between two points along the $z$-axis. These correlations are normalised by a constant $C_{0}=C_{u_{x}}(0)$. Figure 2 shows $C_{u_{x}}(\delta z)$ across various control parameters. Similar behaviour is seen for $C_{u_{x}^{\prime}}(\delta z)$. For $\delta z / H>0.9 C_{u_{x}}(\delta z)$ tends to zero. This is caused by the upper boundary of the tank.

The characteristic length scales are generally determined by finding the first zero in $C_{u_{x}}(\delta z)$. Figure 2 shows that in practice $C_{u_{x}}(\delta z)$ does not necessarily fully decorrelate over $h_{p} \leqslant z \leqslant H$. Hence, following Staplehurst et al. (2008), $l_{z}$ and $l_{z}^{\prime}$ are derived using an arbitrary threshold value $\beta$. At separation distance $Z$, when $C_{u_{x}}(z) / C_{0}=\beta, l_{z}$ is defined using

$$
\ell_{z}=\int_{0}^{Z} \frac{C_{u_{x}}(z)}{C_{0}} \mathrm{~d} z
$$



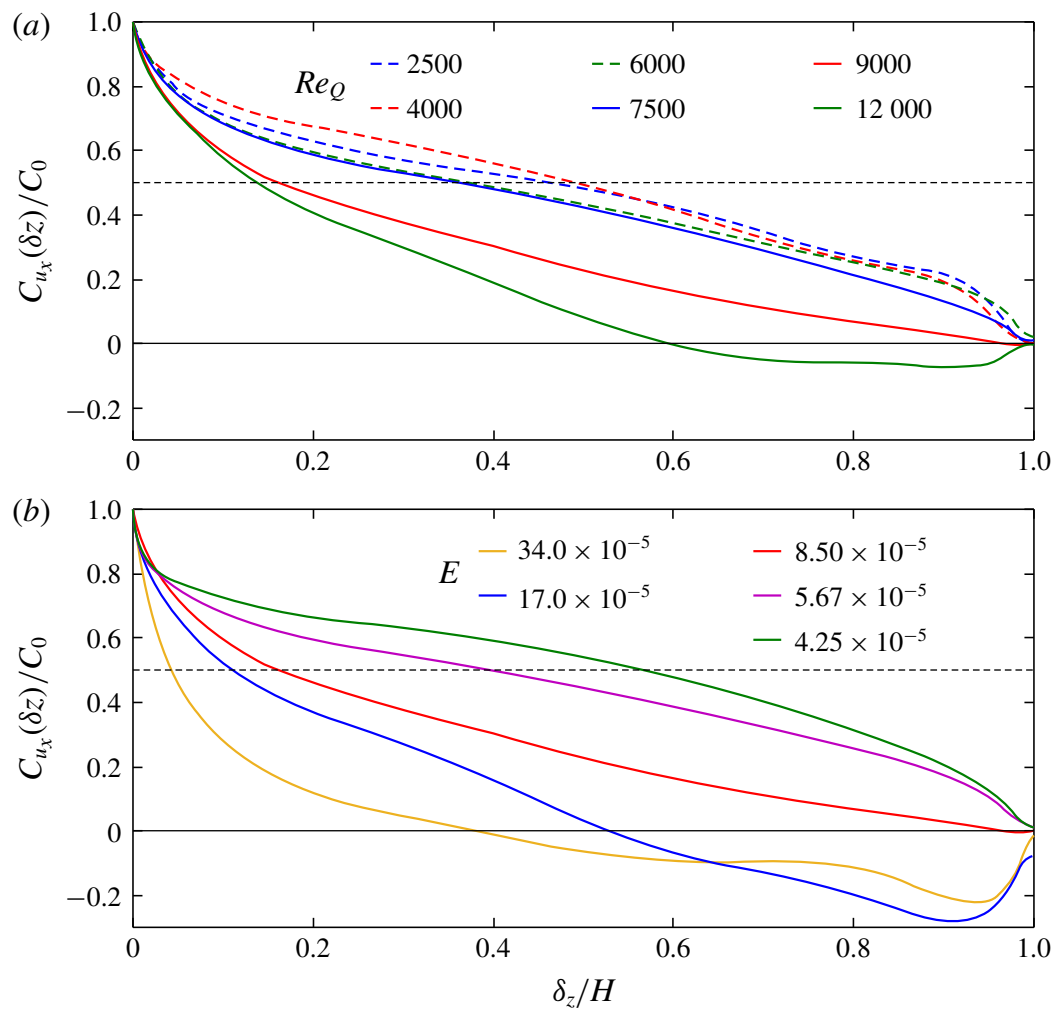

FIGURE 2. Normalised two-point velocity correlations $C_{u_{x}}(\delta z)$ based on separation distance $\delta z$. (a) $C_{u_{x}}$ across various $R e_{Q}$ for $E=8.50 \times 10^{-5}$. (b) $C_{u_{x}}$ for various $E$ at $R e_{Q}=9000$. Dashed black lines represent threshold value $\beta$ used to calculate correlation length $l_{z}$ across all experiments.

As can be seen in figure $2(a, b)$ the behaviour of correlations shift drastically across both $E$ and $R e_{Q}$. Therefore we chose $\beta=0.5$ so as to be able to apply the same method across all experiments.

Due to the limited space across which the correlations can be applied $\left(h_{p} \leqslant z \leqslant H\right)$ and the application of the arbitrary threshold there is scatter in the data for $l_{z}$ and $l_{z}^{\prime}$, as seen in figure 3. Nevertheless, both $l_{z}$ and $l_{z}^{\prime}$ closely follow the $l_{z} \sim L R o^{-1}$ scaling from (2.6). This confirms that columns above the turbulent patch form under the combined influence of the Coriolis forces and inertia. Inertia may, however, be associated with the average flow or to fluctuations, which in turn may be either random or driven by inertial waves. Evaluating the relative importance of the terms in equations (2.7)-(2.9) shall therefore highlight flow regimes where inertial waves are active.

\subsection{Separating inertial waves from turbulent fluctuations}

To this end, we first need to distinguish inertial waves from random turbulent fluctuations. This is done by splitting the turbulent energy spectrum into fluctuations of frequency $f$ greater than the maximum frequency of inertial waves $2 \Omega$ (subscript FT) (Greenspan 1968), and fluctuations of frequency $f<2 \Omega$, which may result from inertial waves or from random turbulence (subscript IW). The ratio of the total energy 


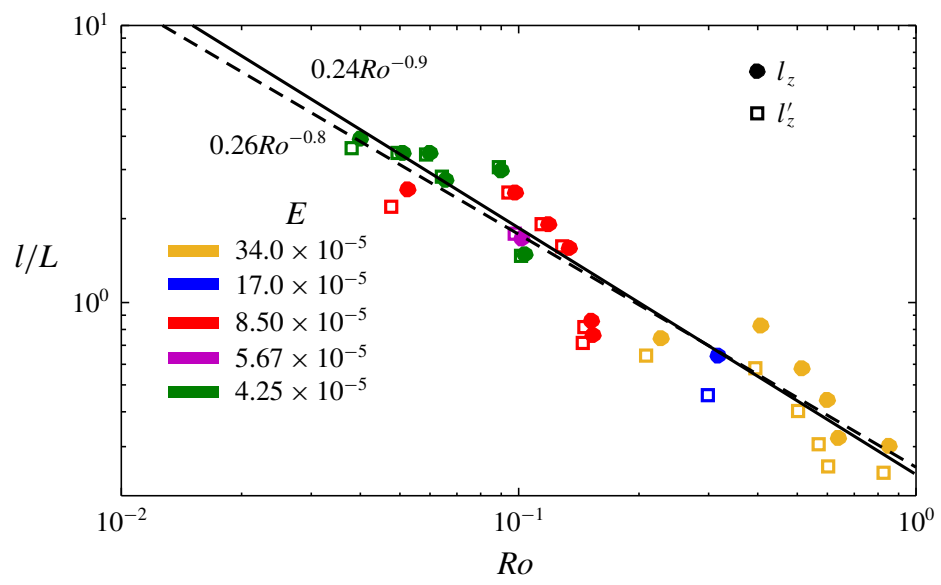

FIGURE 3. Columnar structure length $l_{z}$ based on $u_{x}$ and $l_{z}^{\prime}$ based on $u_{z}^{\prime}$ normalised by $L$. $R o$ based on $|\boldsymbol{u}|$ and $\left|\boldsymbol{u}^{\prime}\right|$ respectively. Solid and dashed black line show a fit of $l_{z}$ and $l_{z}^{\prime}$ data respectively. Data from PIV in the VP.

contained in the lower part of the spectrum $E_{I W}^{\prime}$ to the total energy $E^{\prime}$ provides an upper bound for the fraction of the turbulent kinetic energy carried by inertial waves. Although global, this approach is similar to Campagne et al.'s (2015) scale-dependent disentanglement method. The frequency spectra in figure 4 show that most of the fluctuations' kinetic energy lies within the spectral range of inertial waves provided $R o \gtrsim 10^{-2}$ and $R e_{Q}<4000$. The sharp drop of energy in the spectra precisely at $f=2 \Omega$ (figure $5 a$ ) suggests that the ratio $E_{I W}^{\prime} / E^{\prime}$ reflects the relative importance of inertial waves, at least to some extent. The absence of inertial waves in the higher range of either $R o$ or $R e_{Q}$ reflects their disruption by random turbulence. In freely decaying turbulence, this phenomenon is controlled by the ratio between inertia and the Coriolis force, and takes place at $R o^{\prime} \gtrsim 0.4$ (Staplehurst et al. 2008). Here, inertial waves vanish for $R e_{Q} \gtrsim 10^{4}$, independently of the intensity of the Coriolis force, most likely on the grounds that both the inertial waves and the inertia that disrupt them are driven by fluctuations in the turbulence patch whose intensity is entirely controlled by inertia.

Di Leoni et al. (2014) and Yarom \& Sharon (2014) made similar observations of a spectral energy drop at $f=2 \Omega$, down to lower values of $E$. These authors also found that fluctuations in the range $f<2 \Omega$ obeyed a scaling law $E(f) \propto f^{-1.35}$ consistent with $E(f) \sim(f / 2 \Omega)^{-1.39}$, which we found in the lower frequency range of that region. Unlike these earlier experiments, however, our spectra exhibit an intermediate region $0.2 \lesssim f / 2 \Omega \leqslant 1$, where $E(f)$ does not clearly follow a power law. Since the Rossby number associated with fluctuations in this range is larger than at the lower frequencies, and that the effects of rotation are weaker here than in the regimes of lower Ekman number considered by Yarom \& Sharon (2014), it is likely that this intermediate range of frequencies is significantly affected by inertial effects unlike the range $f / 2 \Omega \lesssim 0.2$, where the Coriolis force dominates.

Next, to better assess how well the $2 \Omega$ frequency separates inertial waves form random fluctuations, we first analyse the flow patterns corresponding to frequencies respectively lower and higher than $2 \Omega$, for $10^{-2} \leqslant R o \leqslant 10^{-1}$. Frequency-specific flow patterns are obtained by applying a pass-band filter centred on a given frequency $f$, 

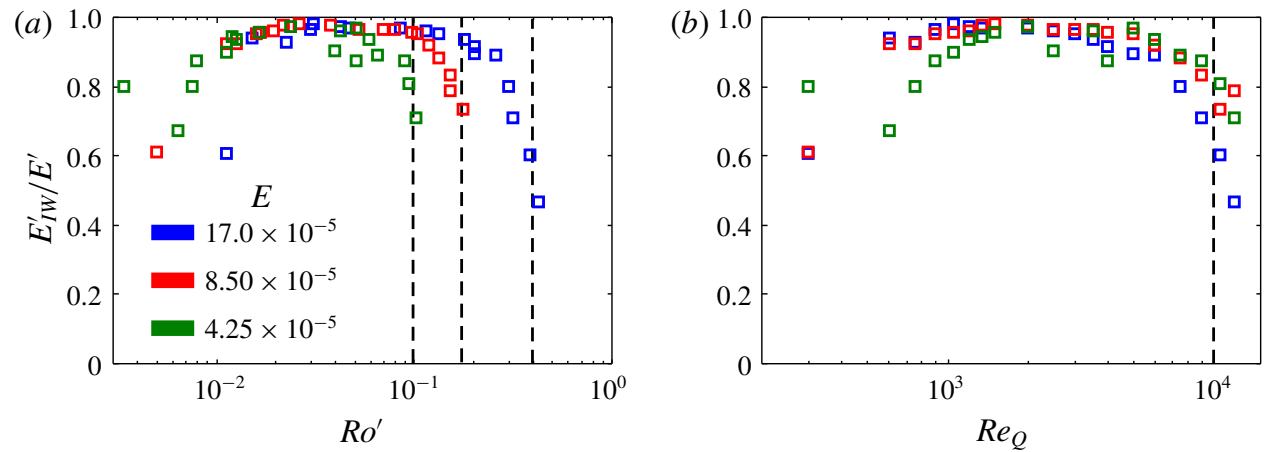

FIGURE 4. Upper bound of energy carried by inertial wave fluctuations to energy carried by the fluctuations versus $(a) R o^{\prime}$ and $(b) R e_{Q}$ for various $E$ at $z=0.75 H$.
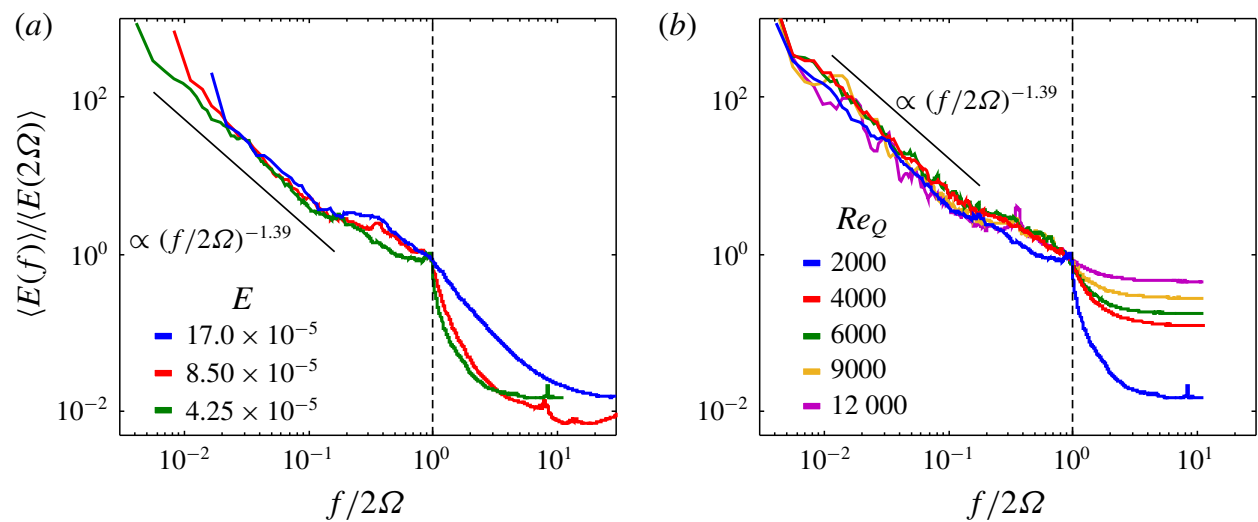

FIgURE 5. Power spectra normalised by $\langle E(2 \Omega)\rangle$ at $(a) R e_{Q}=2000$ with varying $E$ and (b) $E=4.25 \times 10^{-5}$ with varying $R e_{Q}$. Data from PIV in the VP.

to the time-dependent field $\left(u_{x}^{\prime}, u_{z}^{\prime}\right)$, followed by phase averaging, as in Cortet, Lamriben \& Moisy (2010). The bandwidth of the filter $d f$ was kept constant at a range of $d f / 2 \Omega=10^{-2}$. For $f<2 \Omega$, the resulting field reveals individual inertial wave packets being radiated from the turbulent patch (illustrated in figure $6 a-c$ ). These waves propagate throughout the flow field at an angle $\theta$, reflecting at the walls. Since these patterns are detected far enough from the wall, they are nearly axisymmetric on average, so the chevron patterns observed in figure $6(a-c)$ are in fact the two-dimensional signature of the three-dimensional cones observed by Duran-Matute et al. (2013). When $f>2 \Omega$, by contrast, wave-like patterns give way to random fluctuations that remain mostly localised near the turbulent patch (figure $6 d$ ). The propagation angle $\theta$ of the patterns is found by seeking the maximum of two-dimensional spatial cross-correlations of the frequency filtered velocity components $\left(u_{x}^{\prime}, u_{z}^{\prime}\right)$, averaged over time. Figure 6(e-g) shows the correlation patterns for $u_{x}^{\prime}$. Similar patterns are seen for $u_{z}^{\prime}$. This technique reveals patterns with a well-defined propagation angle when $f<2 \Omega$. When $f>2 \Omega$, by contrast, signals only remain well correlated around the origin, confirming that no inertial waves are present in this regime. The relation $\theta(f)$ obtained in this manner is represented in figure 7, and found in very good agreement with the dispersion relation of inertial waves (1.1). 

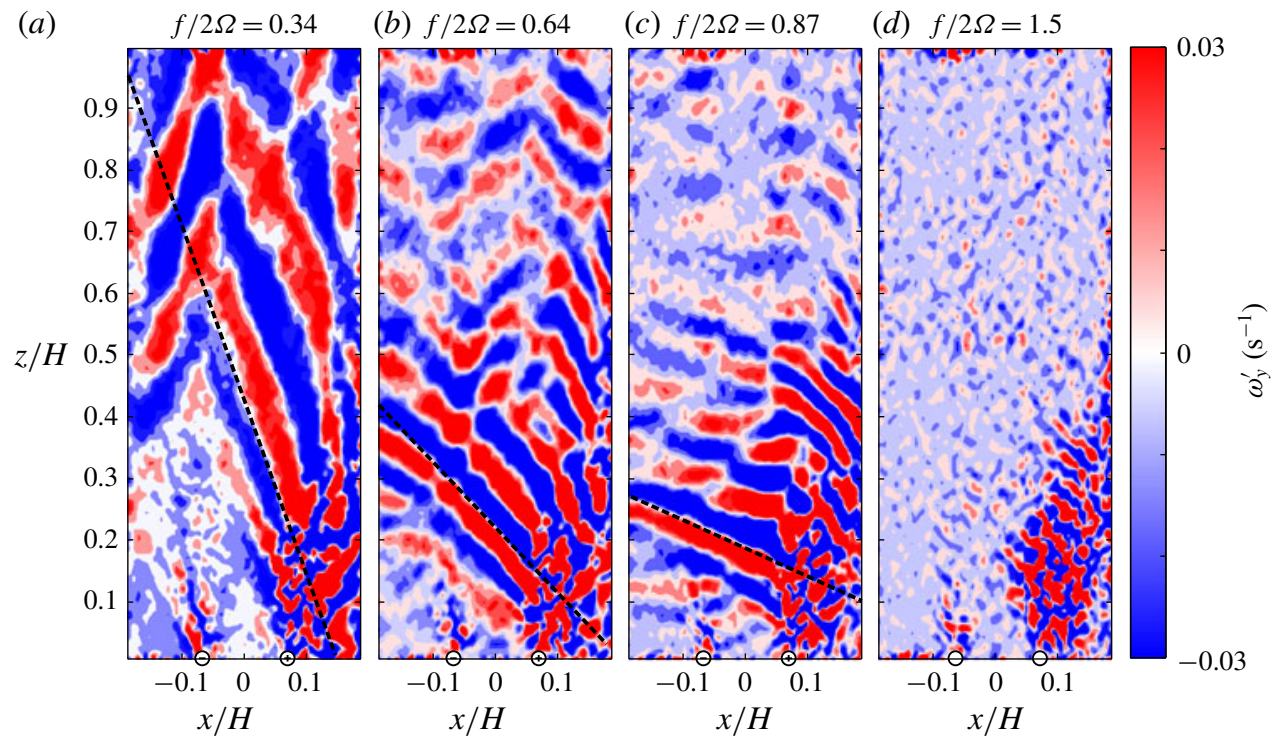

(e)

$(f)$
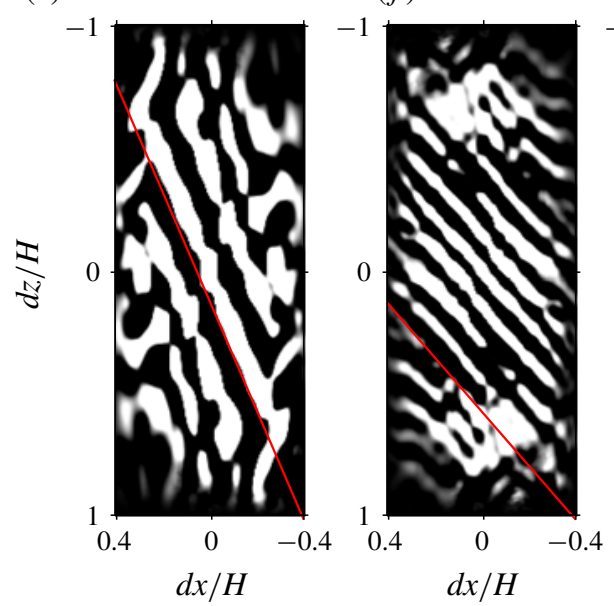

$(g)$

(h)
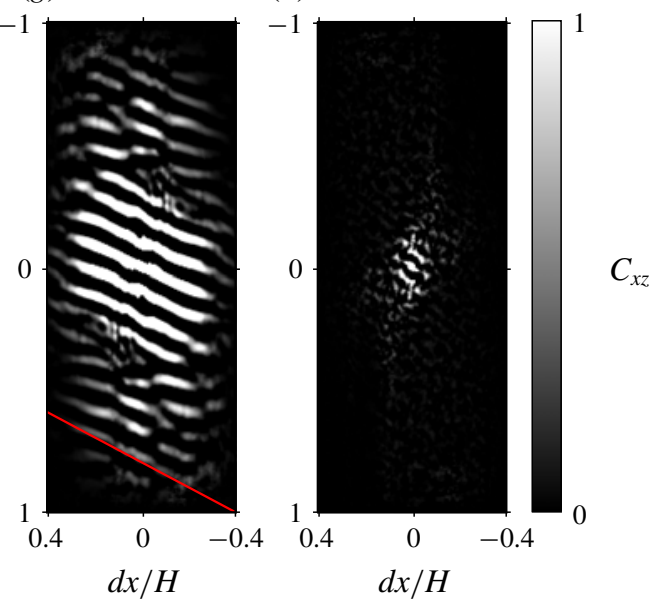

FIGURE 6. $(a-d)$ Filtered vorticity field $\omega_{y}^{\prime}$ at various frequencies $f$. Dashed black lines represent the angle of propagation $\theta$ predicted by the dispersion relation for inertial waves. $(+)$ and $(-)$ represent the approximate locations of the source and sink, respectively. When $f<2 \Omega$ wave-like patterns are found. $(e-h)$ Two-dimensional cross-correlations of $u_{x}^{\prime}$ filtered at frequencies $f$ for $R e_{Q}=1200$ and $E=4.25 \times 10^{-5}$. Red lines represent the propagation angle $\theta$ predicted by dispersion relation for inertial waves.

This confirms that inertial waves are confined in the $f<2 \Omega$ range and that they carry a significant fraction of the fluctuations' energy in this range. Interestingly, the presence of mean advection in the $z$ direction does not incur any Doppler shift.

Finally, to quantify the relevance of the upper bounds for the nonlinear terms obtained by filtering out the contribution of fluctuations of frequency larger than $2 \Omega$, we now need to quantify the fraction of energy carried by inertial waves in the range $f<2 \Omega$. We start with the spectral energy distribution of the fluctuations, represented in a $(k, \theta)$ plane in figure 8 . These spectra are derived using techniques 


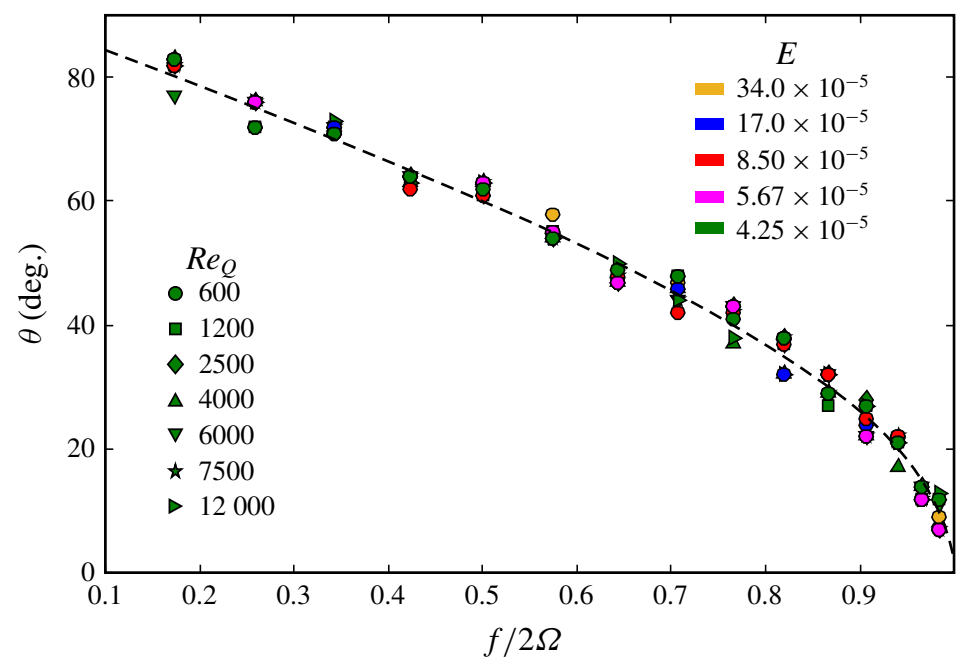

FIgURE 7. Propagation angle $\theta$ of wave-like patterns identified from vorticity fields $\omega_{y}^{\prime}$ filtered at frequency $f$. Dashed black line represent the dispersion relation for inertial waves (Greenspan 1968).

similar to Yarom \& Sharon (2014). In this representation, inertial waves are located near a line defined by their dispersion relation (1.1). Indeed, most of the energy is located there in the limit of low Ekman and Rossby numbers while the lower part of the graph is progressively contaminated as energy of frequency lower than $2 \Omega$ is increasingly carried by random fluctuations. Interestingly, only a very small amount of energy is transferred to evanescent waves of frequency greater than $2 \Omega$ at low $R o$. The fraction of energy $E_{\text {band }}^{\prime} / E_{I W}^{\prime}$ lying within $10 \%$ of the dispersion wave (i.e. $\left|f-f_{I W}(\theta)\right| / f<10 \%$, where $f_{I W}(\theta)$ is the frequency of inertial wave propagating at angle $\theta$ ), is represented in figure 9. As expected, the upper bound captures a greater share of inertial waves in the $R o \rightarrow 0$ limit, and indeed a fair fraction of them up (up to $80 \%$, down to $30 \%$ ) for most of the values of $R o$ investigated. This percentage is, however, only indicative, as it depends directly on how close to the exact dispersion relation fluctuations have to lie to be counted as inertial waves. Nevertheless, the fact remains that the simple filtering technique we employ not only provides a rigorous upper bound for the contribution of inertial waves but also a relevant one in the $R o \rightarrow 0$ limit. As such whenever quantities involving these bounds vanish in this limit, they provide unequivocal evidence that the contribution of inertial waves do. The flip side of using rigorous upper bounds is that they are not indicative of the behaviour of inertial waves for $R o \gtrsim 0.1$, and this shall have to be kept in mind when interpreting our results.

\subsection{Contributions of inertial waves to anisotropy}

We are now in a position to estimate the nature and the magnitude of the contribution to anisotropy within the vertical plane due to fluctuations arising from inertial waves in (2.7)-(2.9). From figure $10(a)$, contributions to the inertial terms in (2.7)-(2.9) are almost strictly due to fluctuations arising from inertial waves for $R o \lesssim 0.1$ at $z=0.38 H$. A similar trend is seen at $z=0.75 H$. Hence, for $R o \ll 0.1$, we assume $\left|\left\langle\boldsymbol{u}^{\prime} \cdot \nabla u_{z}^{\prime}\right\rangle\right|^{e} \approx$ $\left|\left\langle\boldsymbol{u}^{\prime} \cdot \nabla u_{z}^{\prime}\right\rangle\right|_{I W}^{e},\left|\left\langle\boldsymbol{u}^{\prime} \cdot \nabla \omega_{z}^{\prime}\right\rangle\right|^{e} \approx\left|\left\langle\boldsymbol{u}^{\prime} \cdot \nabla \omega_{z}^{\prime}\right\rangle\right|_{I W}^{e}$ and $\left|\left\langle\boldsymbol{\omega}^{\prime} \cdot \nabla u_{z}^{\prime}\right\rangle\right|^{e} \approx\left|\left\langle\boldsymbol{\omega}^{\prime} \cdot \nabla u_{z}^{\prime}\right\rangle\right|_{I W}^{e} \cdot$ 

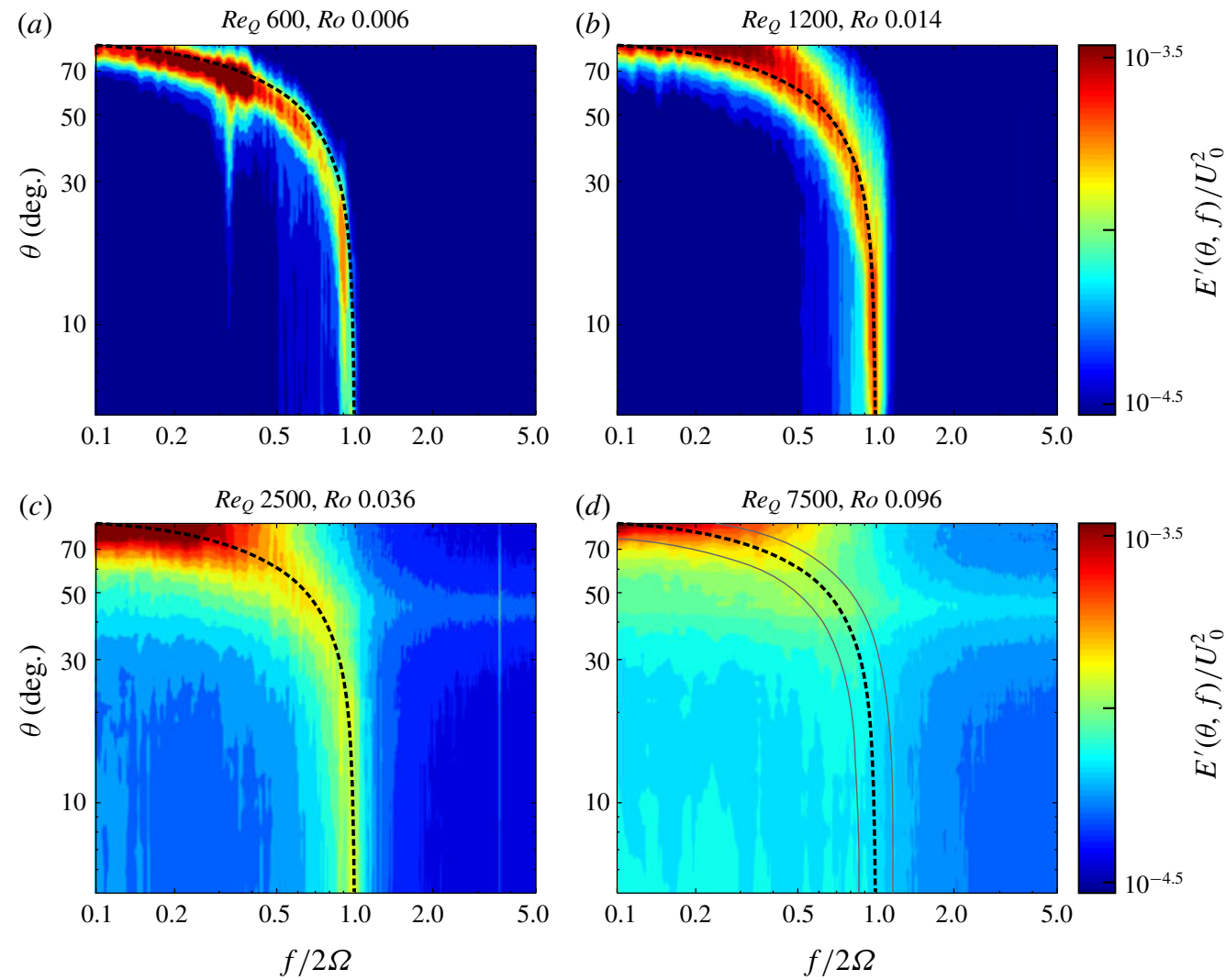

FIGURE 8. Energy density spectra $E^{\prime}(\theta, f)$ at $E=4.25 \times 10^{5}$ for various $R e_{Q}$. Dashed black line shows the dispersion relation for inertial waves. Grey lines in $(d)$ show the narrow band considered to calculate $E_{\text {band }}^{\prime}$.

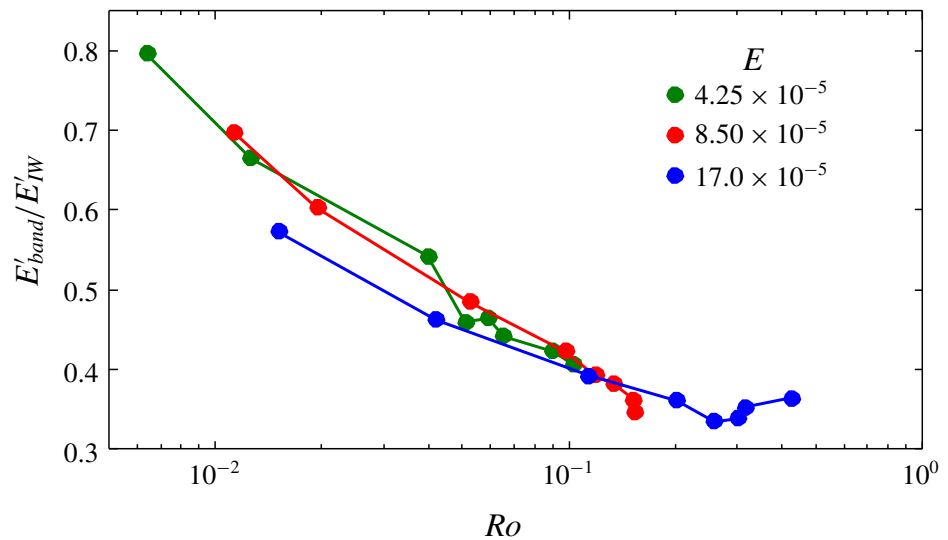

FIGURE 9. Ratio $E_{\text {band }}^{\prime} / E_{I W}^{\prime}$ as function of Ro showing the efficiency of the filtering method applied.

From, figure $10(b)$, the ratio $\left|\left\langle\boldsymbol{u}^{\prime} \cdot \nabla u_{z}^{\prime}\right\rangle\right|_{I W}^{e} /\left|\langle\boldsymbol{u}\rangle \cdot \nabla\left\langle u_{z}\right\rangle\right|$ scales as a positive power of $R o$ (around 2), both in the lower $(z=0.38 H)$ and upper $(z=0.75 H)$ parts of the 
(a)

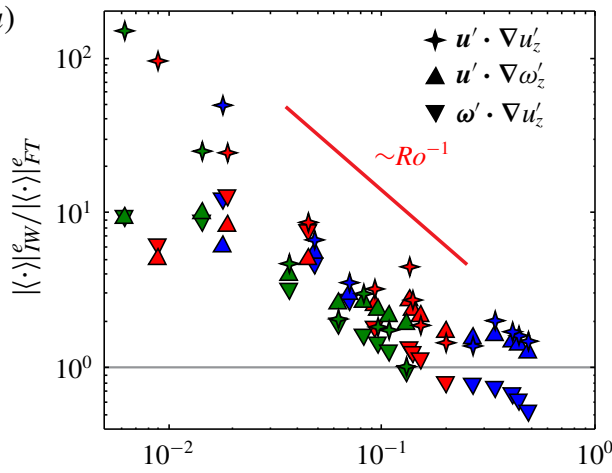

(c)

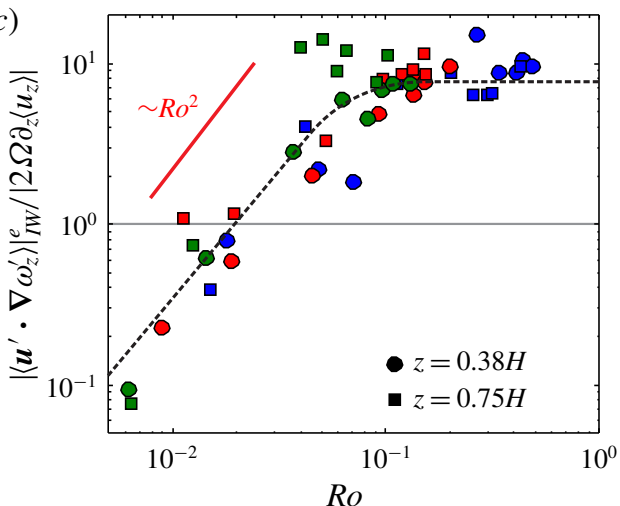

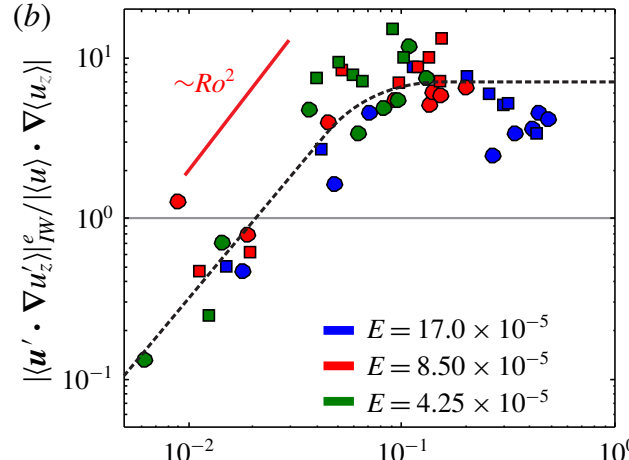

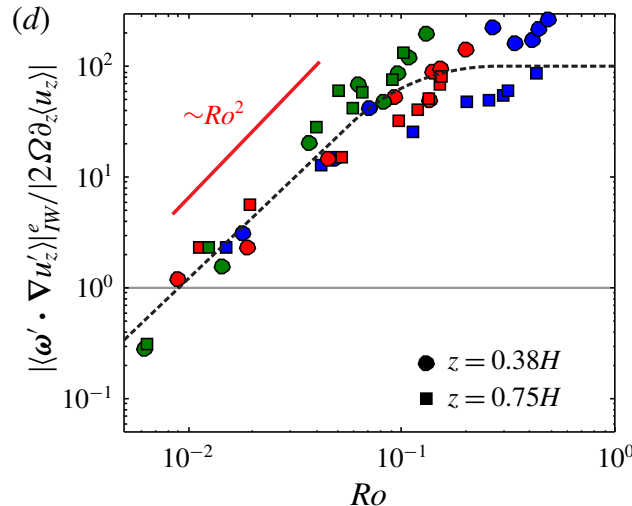

FIgURE 10. (a) Ratio between contributions by inertial waves fluctuations (IW) and random turbulent fluctuations (FT) to inertial terms in (2.7)-(2.9) at $z=0.38 H$. (b) Ratio $\left|\left\langle\boldsymbol{u}^{\prime} \cdot \nabla u_{z}^{\prime}\right\rangle\right|_{I W}^{e} /\left|\langle\boldsymbol{u}\rangle \cdot \nabla\left\langle u_{z}\right\rangle\right|$ at $z=0.38 H$ (circles) and $z=0.75 H$ (squares). (c) Ratio of $\left|\left\langle\boldsymbol{u}^{\prime} \cdot \nabla \omega_{z}^{\prime}\right\rangle\right|_{I W}^{e}$ and $(d)\left|\left\langle\boldsymbol{\omega}^{\prime} \cdot \nabla u_{z}^{\prime}\right\rangle\right|_{I W}^{e}$ to Coriolis term $\left|2 \Omega \partial_{z}\left\langle u_{z}\right\rangle\right|$. Black dashed lines show trend seen across the whole experimental parameter range. Same colour legend across all four graphs. Scalings are indicative only, they do not rely on any theoretical consideration at this stage.

flow for $R o \lesssim 0.15$. For $R o \gtrsim 2 \times 10^{-2}$, the estimate for fluctuations due to inertial waves is greater than inertia due to the average flow. In this regime, however, the estimate includes a significant contribution from random fluctuations (see § 4.2) and is therefore not indicative of the contribution of inertial waves. For $R o \lesssim 2 \times 10^{-2}$, by contrast, this ratio becomes lower than unity and in the limit $R o \rightarrow 0$, fluctuations due to inertial waves cannot balance the pressure gradient in (2.8) so waves play no part in determining $\left\langle u_{z}\right\rangle$. Note that all scalings reported in figure 10 are indicative only, and that while the quantities plotted in all four graphs follow the same exponent in $R o$, there is no theoretical basis to expect that data obtained at different values of $E$ and different heights $H$ collapse into the same curve. Consequently, it follows from the asymptotic behaviour in the limit $R o \rightarrow 0$, and from (2.8) and (2.10) that

$$
\langle\boldsymbol{u}\rangle \cdot \nabla\left\langle u_{z}\right\rangle=2 \Omega \Delta^{-1} \partial_{z}\left\langle\omega_{z}\right\rangle+\mathrm{FT}+O(R o),
$$

where FT stands for any term involving fluctuations not due to inertial waves. Consequently, if inertial waves are to influence the anisotropy of the mean flow, 
they can only do so through the last two terms of (2.7). Their part in this process is measured by the ratio of their contribution to inertial terms $\left|\left\langle\boldsymbol{u}^{\prime} \cdot \nabla \omega_{z}^{\prime}\right\rangle\right|$ and $\left|\left\langle\boldsymbol{\omega}^{\prime} \cdot \nabla u_{z}^{\prime}\right\rangle\right|$ (whose estimates are denoted $\left|\left\langle\boldsymbol{u}^{\prime} \cdot \nabla \omega_{z}^{\prime}\right\rangle\right|_{I W}^{e}$ and $\left|\left\langle\omega^{\prime} \cdot \nabla u_{z}^{\prime}\right\rangle\right|_{I W}^{e}$ ) to the Coriolis term $\left|2 \Omega \partial_{z}\left\langle u_{z}\right\rangle\right|$. From figure $10(c, d)$, the variations of these ratios with $R o$ exhibit the same two regimes singled out in the analysis of (2.8). For $R o \gtrsim 2 \times 10^{-2}$, they are greater than unity and grow until they saturate at values between 10 and $10^{2}$. The saturation coincides with the regimes where inertial waves are superseded by random fluctuations (see figures 4 and $10 a$ ), and marks the end of the rotation-dominated turbulent regime. Again, estimates are not representative of the behaviour of inertial waves in this range. Below the saturation and for $R o \gtrsim 0.2$, on the other hand, inertial waves dominate and (2.7) chiefly expresses a transfer of energy between them and the mean flow. For $R o \lesssim 2 \times 10^{-2}$ and in the limit $R o \rightarrow 0$, the mean Coriolis term dominates in (2.7) and from (4.3), it directly determines the flow anisotropy in the vertical plane according to

$$
(\langle\boldsymbol{u}\rangle \cdot \nabla)^{2}\left\langle\omega_{z}\right\rangle-\langle\boldsymbol{u}\rangle \cdot \nabla\left(\langle\boldsymbol{\omega}\rangle \cdot \nabla\left\langle u_{z}\right\rangle\right) \simeq 4 \Omega^{2} \partial_{z z}^{2} \Delta^{-1}\left\langle\omega_{z}\right\rangle-2 \Omega \partial_{z}\langle\boldsymbol{u}\rangle \cdot \nabla\left\langle u_{z}\right\rangle+\mathrm{FT} .
$$

\section{Conclusion and discussion}

Equation (4.4) establishes our main result: in the limit $R o \rightarrow 0$ inertial waves are not part of the mechanism driving the anisotropy of the average turbulent flow in background rotation. The actual mechanism involves a balance between advection of the average flow and the Coriolis force. From this point of view, it also differs from the diffusive process underpinning the formation of columns in Taylor's experiment. In both cases, however, anisotropy is materialised by a horizontally divergent flow either driven by inertia (turbulent flows) or by viscous friction (Taylor columns).

The physical mechanism governing the anisotropy of the average flow along the rotation axis is fully captured by (4.4), but more conveniently traced from the set of averaged equations (2.7)-(2.9), stripped from the contributions of inertial waves. As for the full flow field, the average flow $\langle\boldsymbol{u}\rangle$ is columnar in the limit $\Omega \rightarrow \infty$. Here, however, the departure to two-dimensionality for the average flow arises out of the inertial terms in (2.7), which presents itself in two forms: one is driven by inertia of the average flow itself (terms involving averages only), the other by inertia associated with fluctuations. Since we now know the contribution of inertial waves to these terms to be small, compared to the dominant Coriolis term in the limit $R o \rightarrow 0$, the divergent flow has to be predominantly driven by the other contributions to inertia: either from the average flow, or from random turbulent fluctuations. In turn, the vertical flow sustained by this mechanism, drives an average vertical pressure gradient through (2.8), and subsequently, a $z$-dependence on the vertical vorticity component through (2.9), that defines its anisotropy.

This mechanism acts in two ways: the presence of inertia away from Ekman boundaries drives a departure to two-dimensionality through the secondary flows, with a mediating role played by the pressure. Conversely, if a localised horizontal force is applied to drive the flow (adding an inhomogeneous forcing term $\nabla \times f$ in (2.7)), then an average secondary flow with a vertical velocity component $\partial_{z}\left\langle u_{z}^{f}\right\rangle=-(1 / 2 \Omega) \nabla \times f$ is driven by the Coriolis force to be able to balance the rotational part of $f$. Inertia then diverts the vertical flow into secondary flows, up to a distance $l_{z}$ from the location of the forcing, where the vertical flow driven by the forcing is exhausted. This mechanism controls the vertical length scale (and anisotropy) of the average length scale set in motion by $\boldsymbol{f}$, as expressed by scaling (2.6). 
This mechanism explains how average anisotropy can develop along the rotation axis without the involvement of inertial waves. It is closely related to the phenomenon of inertial mixing put forward by McEwan (1976), but also generic in current-driven flows, whether this current involves mass or electric charge (in MHD flows, see Pothérat (2012), Pothérat \& Klein (2017)). In magnetohydrodynamic (MHD) flows within the quasi-static approximation, Alfvén waves, which are the MHD counterpart of inertial waves, are altogether absent. Anisotropy develops through a diffusive process, in which the electric potential plays a very similar mediating role to that played by the pressure in rotating flows (Pothérat, Sommeria \& Moreau 2000). Similarly, electric current is driven along the magnetic field lines for the Lorentz force to balance the rotational part of inhomogeneous forces and the point where inertial forces have exhausted the current produced by them determines the length scale of the flow structure set in motion (Pothérat \& Klein 2017).

Finally, it is noteworthy that in the limit $R o \rightarrow 0, l_{z}^{I}\left(l_{\perp}\right) / H \rightarrow \infty$ for all scales $l_{\perp}$, and the flow becomes quasi-two-dimensional. Hence, our main result also implies that inertial waves are not responsible for the two-dimensionalisation of the average flow either. Thus the new mechanism argued for here may explain the formation of Taylor columns in average flows at high Reynolds numbers. We insist, however, that it does not concern Taylor columns found in the fluctuating part of the flow. In particular, flows with nil average flow, such as randomly forced turbulent flows in three-dimensional periodic domains, may admit a basis of inertial modes. This implies that the formation of the fluctuating columns in these flows involves interactions between these waves, following either of the mechanisms proposed by Smith \& Waleffe (1999). By contrast, where a non-zero average exists, any basis must involve non-oscillatory modes (such as the leading-order zonal flow in a sphere (Greenspan 1968)). The experimental configuration studied in this paper falls in this category and it is precisely this steady component that is governed by the waveless mechanism we put forward. As such, this result does not exclude that in complex turbulent flows, the anisotropy of the average component may be driven by the waveless mechanism while at the same time anisotropic turbulent structures may be shaped by inertial waves.

\section{Acknowledgements}

The authors would like to acknowledge support from the Engineering and Physical Sciences Research Council (grant number GR/N64519/01) for the manufacture of the rotating turntable facility and B. Teaca for computational resources used in processing experimental data. A.P. acknowledges support from the Royal Society under the Wolfson Research Merit Award Scheme (Grant reference WM140032).

\section{REFERENCES}

Aujogue, K., Pothérat, A., B., S. \& F., D. 2018 Experimental study of the convection in a rotating tangent cylinder. J. Fluid Mech. 843, 355-381.

Baker, N., Pothérat, A., Davoust, L. \& Debray, F. 2018 Inverse and direct energy cascades in three-dimensional magnetohydrodynamic turbulence at low magnetic Reynolds number. Phys. Rev. Lett. 120, 224502.

Brons, J. A., Thomas, P. J. \& Pothérat, A. 2020 Transition between advection and inertial wave propagation in rotating turbulence. J. Fluid Mech. 886, A22.

CAmbon, C. \& ScotT, J. F. 1999 Linear and non-linear models in anisotropic turbulence. Аnnu. Rev. Fluid Mech. 31, 1-53. 
Campagne, A., Gallet, B., Moisy, F. \& Cortet, P. P. 2015 Disentangling inertial waves from eddy turbulence in a forced rotating-turbulence experiment. Phys. Rev. E 91, 043016.

Campagne, A., Gallet, B., Moisy, F. \& Cortet, P. P. 2014 Direct and inverse energy cascades in a forced rotating turbulence experiment. Phys. Fluids 26, 125112.

Chen, Q., Chen, S., Eyink, G. L. \& Holm, D. D. 2005 Resonant interactions in rotating homogeneous three-dimensional turbulence. J. Fluid Mech. 542, 139-164.

Cortet, P. P., LAmriben, C. \& Moisy, F. 2010 Viscous spreading of an inertial wave beam in a rotating fluid. Phys. Fluids 22, 086603.

Davidson, P. A. 2013 Turbulence in Rotating, Stratified and Electrically Conducting Fluids. Cambridge University Press.

Davidson, P. A., Staplehurst, P. J. \& Dalziel, B. 2006 On the evolution of eddies in a rapidly rotating system. J. Fluid Mech. 557, 135-144.

Di Leoni, P. C., Cobelli, P. J., Mininni, P. D., Dmitruk, P. \& Matthaeus, W. H. 2014 Quantification of the strength of inertial waves in a rotating turbulent flow. Phys. Fluids 26, 035106.

DiCKINSON, S. C. \& LONG, R. R. 1983 Oscillating-grid turbulence including effects of rotation. J. Fluid Mech. 126, 315-333.

Duran-Matute, M., Flór, J.-B., Godeferd, F. S. \& Jause-Labert, C. 2013 Turbulence and columnar vortex formation through inertial-wave focusing. Phys. Rev. E 87, 041001.

GALLET, B. 2015 Exact two-dimensionalization of rapidly rotating large-Reynolds-number flows. J. Fluid Mech. 783, 412-447.

Greenspan, H. P. 1968 The Theory of Rotating Fluids. Cambridge University Press.

Hopfinger, E. J., Browand, F. K. \& Gagne, Y. 1982 Turbulence and waves in a rotating tank. J. Fluid Mech. 125, 505-534.

Kolvin, I., Cohen, K., Vardi, K. \& Sharon, E. 2009 Energy transfer by inertial waves during the buildup of turbulence in a rotating system. Phys. Rev. 102, 014503.

Lamriben, C., Cortet, P. P. \& Moisy, F. 2011 Direct measurements of anisotropic energy transfers in a rotating turbulence experiment. Phys. Rev. Lett. 107, 024503.

MAXWORTHY, T. \& NARIMOUSA, S. 1994 Unsteady, turbulent, covection into a homogeneous, rotating fluid with oceanographic applications. J. Phys. Oceanogr. 24, 865-887.

MCEWAN, A. D. 1973 A laboratory demonstration of anglular momentum mixing. Geophys. Fluid Dyn. 5, 283-311.

McEwan, A. D. 1976 Angular momentum diffusion and the formation of cyclones. Nature 260, $126-128$.

Moore, D. W. \& SAfFman, P. G. 1969 The flow induced by the transverse motion of a thin disk in its own plane through a contained rapidly rotating viscous liquid. J. Fluid Mech. 39 (4), 831-847.

Pothérat, A. 2012 Three-dimensionality in quasi-two dimensional flows: recirculations and Barrel effects. Eur. Phys. Lett. 98, 64003.

Pothérat, A. \& Klein, R. 2014 Why, how and when MHD turbulence at Low Rm becomes three-dimensional. J. Fluid Mech. 761, 168-205.

Pothérat, A. \& Klein, R. 2017 Do static magnetic fields enhance turbulence at low magnetic Reynolds number? Phys. Rev. Fluids 2 (6), 063702.

Pothérat, A., Sommeria, J. \& Moreau, R. 2000 An effective two-dimensional model for MHD flows with tranverse magnetic field. J. Fluid Mech. 424, 75-100.

Screenivasan, B. \& Davidson, P. A. 2008 On the formation of cyclones and anticyclones in a rotating fluid. Phys. Fluids 20, 085104.

Smith, L. M., Chasnov, J. R. \& WalefFe, F. 1996 Crossover from two- to three-dimensional turbulence. Phys. Rev. Lett. 77 (12), 2467-2470.

Smith, L. M. \& Waleffe, F. 1999 Transfer of energy to two-dimensional large scales in forced, rotating three-dimensional turbulence. Phys. Fluids 11, 1608-1622.

Sommeria, J. \& MoreaU, R. 1982 Why, How and When MHD turbulence becomes two-dimensional. J. Fluid Mech. 118, 507-518. 
Staplehurst, P. J., Davidson, P. A. \& Dalziel, B. 2008 Structure formation in homogeneous freely decaying rotating turbulence. J. Fluid Mech. 598, 81-105.

Tabeling, P. 2002 Two-dimensional turbulence: a physicist approach. Phys. Rep. 362, 1-62.

TAYlor, G. I. 1922 The motion of a sphere in a rotating liquid. Proc. R. Soc. Lond. A 102, $180-189$.

Thomson, J. 1868 Letter to Lord Kelvin (W. Thomson). Glasgow University Library.

YAROM, E. \& SHARON, E. 2014 Experimental observation of steady inertial wave turbulence in deep rotating flows. Nat. Phys. 10 (7), 510-514. 